\title{
Using Social Media for Biosurveillance: Gap between Research and Action
}

\author{
Tera Reynolds*1, Mark Cameron², Mike Conway ${ }^{3}$, Amy Ising ${ }^{4}$, Eric H.Y. Lau ${ }^{5}$, Jennifer \\ Olsen $^{6}$, Julie Pavlin ${ }^{7}$, Bill Storm ${ }^{8}$, Katie Suda ${ }^{9}$ and Courtney Corley ${ }^{10}$
}

${ }^{1}$ International Society for Disease Surveillance, Boston, MA, USA; ${ }^{2}$ Commonwealth Scientific and Industrial Research Organisation, Acton, ACT, Australia; ${ }^{3}$ University of California, San Diego, San Diego, CA, USA; ${ }^{4}$ University of North Carolina-Chapel Hill, Chapel Hill, NC, USA; ${ }^{5}$ University of Hong Kong, Hong Kong, China; ${ }^{6}$ Skoll Global Threats Fund, San Francisco, CA, USA; ${ }^{7}$ Armed Forces Health Surveillance Center, Silver Spring, MD, USA; ${ }^{8}$ Ohio Department of Health, Columbus, OH, USA; ${ }^{2}$ University of Tennessee Health Science Center, Knoxville, TN, USA; ${ }^{10}$ Pacific Northwest National Laboratory, Kennewick, WA, USA

\section{Objective}

The objective of this study is to systematically review the literature on the use of social media for biosurveillance in order to evaluate whether this data source can improve public health practice or community health outcomes.

\section{Introduction}

There is a significant body of literature on the use of social media for monitoring ailments such as influenza-like illness ${ }^{1}$ and cholera, ${ }^{2}$ as well as public opinions on topics such as vaccination. ${ }^{3}$ In general, these studies have shown that social media correlates well with official data sources, ${ }^{1,2,3}$ with the trends identifiable before official data are available. ${ }^{2}$ However, less is known about the impact of integrating social media into public health practice, and resulting interventions. Therefore, the ISDS Social Media for Disease Surveillance Workgroup initiated a systematic literature review on the use of social media for actionable biosurveillance.

\section{Methods}

For the purposes of this project, social media was defined as internet-based applications where users can: activate and set their own profile, develop and update it constantly, make such profile totally or partially public- linking it with other profiles on a network- and share resources and information. Examples of included social media are: Facebook, microblogs, blogs and discussion forums. Data sources such as internet searches and ProMED-mail were not included.

The PRISMA standard ${ }^{4}$ for systematic literature reviews was followed, and Pubmed, Embase and Scopus databases were queried between September 2012 and February 2013 for journal articles, conference proceedings and gray literature. Over 1,440 unique articles were identified and briefly reviewed for relevance to the following questions by at least two reviewers:

1. Can social media be integrated into biosurveillance practice and outbreak management in order to support and improve public health?

2. Can social media be used to effectively target populations, specifically vulnerable populations, in order to test an intervention and interact with a community to improve health outcomes?

Articles were excluded when: neither question was addressed, methods were scientifically unsound or methods were presented exclusively. Review articles were also excluded throughout, although citation lists were searched for additional manuscripts.

At the time of submission this project is in progress, with 231 articles identified for detail review. Of these, 184 have been reviewed using the structured data abstraction form (preliminary results below). The project will be concluded in October, and the final results presented at the ISDS Conference.

\section{Results}

The preliminary results include 29 articles that address the first review question and 23 articles the second. It is worth noting that $89 \%$ of the included articles are classified as exploratory. In general, these tend to be retrospective or survey-based analyses assessing the validity of using social media for monitoring events and sentiments of public health interest, as well as for targeting populations. There are very few articles that discuss social media being integrated in public health practice, and even fewer that include subsequent intervention.

Of the excluded articles, 19 discussed methods exclusively, and in a more developed stage, may address a review question.

\section{Conclusions}

The research questions were focused on the use of social media to support public health practice, and, based on preliminary results, there is little literature on this topic. This may suggest that it is particularly challenging to translate research using social media for biosurveillance into practice. Alternatively, public health departments may be using social media, but not publishing their efforts.

\section{Keywords}

Social media; Literature review; Actionable

\section{Acknowledgments}

This work was supported by ISDS. The authors thank Christine Noonan at PNNL for project support.

\section{References}

1. Achrekar H, Gandhe A, Lazarus R, et al. Twitter improves seasonal influenza prediction. In: HEALTHINF 2012 - Proc of the Intl Conf on Heal Inform. SciTePress; 2012:61-70.

2. Chunara R, Andrews JR, Brownstein JS. Social and news media enable estimation of epidemiological patterns early in the 2010 Haitian cholera outbreak. Am J Trop Med Hyg. 2012;86:39-45.

3. Salathé M, Khandelwal S. Assessing Vaccination Sentiments with Online Social Media: Implications for Infectious Disease Dynamics and Control. PLoS Comput Biol. 2011;7(10):e1002199.

4. PRISMA. http://www.prisma-statement.org/.

\section{*Tera Reynolds}

E-mail: treynolds@syndromic.org 